\title{
МЕТОДИЧЕСКИЕ АСПЕКТЫ ОБУЧЕНИЯ МАТЕМАТИКЕ НЕСПОСОБНЫХ ШКОЛЬНИКОВ
}

\author{
А. Ю. Пигарев
}

Новосибирский государственный университет экономики и управления, Новосибирск

Описаны сущность и причина неспособности к усвоению математике - недостаток рабочей памяти. Охарактеризованы два основных подхода к решению проблемы обучения математике неспособных школьников: 1) тренировка рабочей памяти, 2) снижение нагрузки на рабочую память в учебном процессе. Отмечена неоднозначность результатов внедрения первого подхода: тренировка рабочей памяти приводит к улучшению результатов прохождения тестов на рабочую память, однако может не приводить к улучшению показателей обучаемости, связанных с рабочей памятью ученика. Этим обосновывается приоритет второго подхода. Представлены как уже известные методы снижения нагрузки на рабочую память при обучении математике, так и разработанные автором компьютерные тренажеры, направленные на автоматизацию базовых вычислительных навыков (арифметических, тригонометрических, геометрических). Дана методика работы с тренажерами на основе метода интервальных повторений и приведены эмпирические данные по результатам их внедрения.

Ключевые слова: математические способности, обучение математике, рабочая память, компьютерный тренажер, интервальные повторения.

Школьники делятся в зависимости от уровня проявления способностей к изучению математики на «очень способных», «способных», «средних» и «неспособных» [1, с. 191]. Как быть с неспособными учениками? В мире цифровой экономики без знаний основ математики человек не может быть успешным ни в одной сфере. Необходимо, поняв причину неспособностей к математике, разработать такие методики обучения, чтобы ребята с плохой успеваемостью могли выйти из замкнутого круга неудач и обрести потенциал роста и развития.

\section{Сущность неспособностей к усвоению математики}

Различия в математических способностях у разных учеников проявляются в том, что «одинаковые упражнения, одинаковая практика, одинаковый опыт (насколько об этой одинаковости можно судить) у школьников с различными способностями дают различные результаты. У одного (его мы и называем способным) эти упражнения приводят к выработке способности к аналитико-синтетическому „видению“, у другого - нет [1, с. 252-253]».

Характеризуя неспособных к математике школьников, В. А. Крутецкий [1] отмечает: «Изучение математики дается таким учащимся с большим трудом, несмотря на их старание и усердие; они не могут рассчитывать на большой успех в математической деятельности, как в смысле быстроты продвижения, так и в смысле уровня достижений [1, с. 189]». «Математические навыки у них формируются с трудом, при большом числе упражнений и являются непрочными - легко распадаются при отсутствии упражнений» [1, с. 190].

Что касается возраста, в котором можно говорить о наличии или отсутствии математических способностей, то, согласно В. А. Крутецкому, способности к математике, так же как и отсутствие таковых, начинают проявляться в VI-VII классах в связи с началом 
систематического изучения алгебры и геометрии, а в IX-X классах способности в каком-то смысле уже сложившееся образование [1, с. 101-102].

Причина неспособности к математике в работе В. А. Крутецкого не раскрыта. Лишь констатация факта: «...неспособность к математике... имеет своей первопричиной большую затрудненность выделения мозгом раздражителей типа математических обобщенных отношений, функциональных зависимостей, числовых абстрактов и символов и затрудненность операций с ними» [1, с. 390-391].

В рамках компьютерной метафоры возникла концепция рабочей памяти человека, основоположником которой был А. Бэддели в конце XX в. [2].

Рабочая память - это система, в которой комбинируется и преобразуется ограниченный объем информации, полученной от органов восприятия, кратковременной и долговременной памяти. Рабочая память поддерживает мыслительные процессы, является интерфейсом между кратковременной и долговременной памятью человека, обеспечивает как процесс консолидации долговременной памяти, так и процесс извлечения из нее информации [2].

Рабочая память человека состоит из центрального администратора и двух хранящих преобразуемую информацию подсистем: зрительно-пространственного блокнота и фонологической (артикуляционной) петли (рис. 1) в соответствии с основной моделью, предложенной Аланом Бэддели [2].

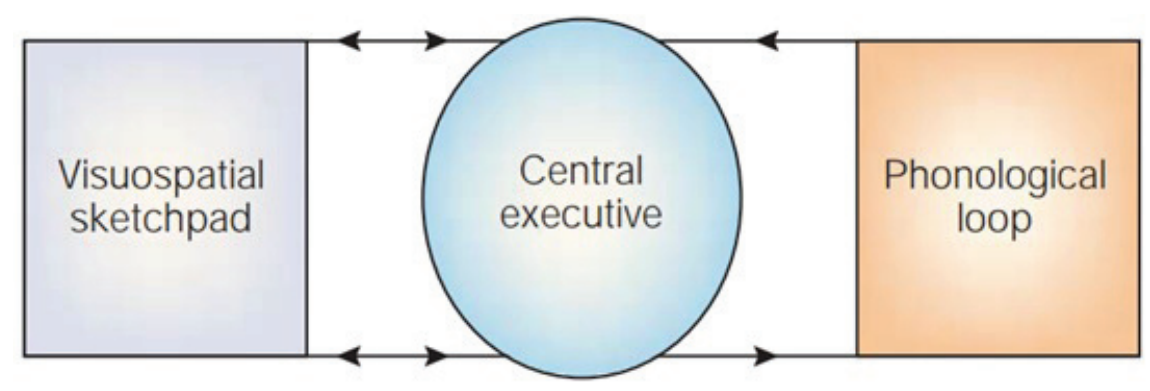

Рис. 1. Трехкомпонентная модель рабочей памяти [2]

Четвертый компонент - эпизодический буфер - был добавлен в модель немного позже. Он отвечает за взаимодействие зрительно-пространственного блокнота и артикуляционной петли с долговременной памятью [2].

Оказалось, что объем рабочей памяти высоко коррелирует с результатами обучения школьников, в особенности математическим дисциплинам: «...memory skills uniquely predicted mathematical skills and arithmetical abilities» [3, c. 136]. Данный вывод был подтвержден множеством других исследований, например [4-7].

Таким образом, именно дефицит рабочей памяти - причина неспособности к изучению математики. Все три компонента основной модели рабочей памяти важны для понимания математики. Математические способности наиболее высоко коррелируют со зрительнопространственным блокнотом, который является как бы мысленной доской, на которой представлены числа и переменные в процессе вычисления и сравнения $[4,5]$. Центральный администратор и фонологическая петля определяют способности к решению вербальных математических задач [6].

На основе концепции рабочей памяти было выработано два подхода к решению проблемы обучения математике неспособных школьников. 


\section{Основные подходы к решению проблемы обучения математике неспособных школьников}

Первый подход - попробовать улучшить рабочую память ученика путем ее тренировки или при обращении за медицинской помощью, если причиной дефицита рабочей памяти является какое-либо заболевание, например когда-то имевшее место сотрясение мозга.

Что касается улучшения рабочей памяти с помощью компьютерных тренажеров, то первые результаты были весьма многообещающими, однако последующие исследования поставили их под сомнение.

Первая работа появилась в 2008 г. [8]. Ее авторы, S. M. Jaeggi, M. Buschkuehl, J. Jonides, W. J. Perrig, утверждали, что тренировка рабочей памяти человека на компьютерных тренажерах, построенных на основе задачи n-back, позволяет за относительно короткий срок (10-20 дней) не только повысить эффективность рабочей памяти, но и развить способность к обучению и усвоению новых знаний. Авторы «представили доказательство переноса результатов тренировки рабочей памяти на развитие подвижного интеллекта» [8]. По их данным, чем больше и продолжительнее были тренировки, тем более заметным было улучшение подвижного интеллекта, оцениваемого с помощью специальных тестов.

Данный вывод опровергается в работе D. L. Dunning, J. Holmes, S. E. Gathercole [9]. Тренировка рабочей памяти строилась на тренажерах от компании Cogmed (https://www. cogmed.com/). Цикл тренировок состоял из 20-25 занятий. Каждое занятие длилось от 30 до 45 мин и включало восемь упражнений. Каждое упражнение повторялось 15 раз. Основатель этой компании профессор Т. Клингберг (Torkel Klingberg, MD, PhD Professor in Cognitive Neuroscience Karolinska Institutet) опубликовал 62 статьи и две научно-популярные книги о пользе созданных им тренажеров (http://www.klingberglab.se/torkel-klingberg/).

Оценивалась рабочая память с помощью стандартизованной тестовой батареи Automated Working Memory Assessment (AWMA) [9]. Данная тестовая система создавалась одним из авторов работы [9] S. E. Gathercole. Упражнения от компании Cogmed содержательно отличаются от заданий тестовой системы AWMA, поэтому авторы работы [9] утверждают, что тренировка рабочей памяти улучшает ее показатели, измеряемые с помощью «нетренированных» тестов: «adaptive WM training significantly boosted performance on untrained WM tasks in children with low WM» [9].

Способность к обучению измерялась с помощью двух тестовых батарей: the Wechsler Abbreviated Scales of Intelligence (WASI) и Kaufman Test of Educational Attainment (KTEA). Улучшение показателей рабочей памяти по результатам выполнения «нетренированных» тестов не привело к улучшению показателей обучаемости по результатам выполнения тестов интеллекта, что позволило авторам работы [9] сделать вывод о бесполезности тренировки рабочей памяти как инструмента повышения успеваемости в учебе. В этом исследовании участвовало 810 школьников.

$\mathrm{C}$ другой стороны, о положительном влиянии тренировки рабочей памяти на успеваемость по математическим дисциплинам утверждается в исследовании [10]. В этом исследовании было задействовано 104 школьника.

В обоих исследованиях участвовали школьники одинаковой возрастной группы (813 лет). Отличались условия проведения исследования. В эксперименте, представленном в работе [9], тренировки проводились в специальной лаборатории, в исследовании [10] - в школе, в которой учились дети. Отличались и программы тренировок. В исследовании [10], помимо классических тренажеров рабочей памяти, были упражнения на развитие вычислительных навыков. 
В настоящее время вопрос о пользе специальной тренировки рабочей памяти остается открытым и дискуссионным. Есть множество работ, в которых утверждается польза от таких тренировок, и не меньше работ, в которых эффективность такого подхода ставится под сомнение.

Второй подход - снижение нагрузки на рабочую память в процессе обучения. Недостаток рабочей памяти не позволяет школьнику воспринимать новый материал, если нагрузка на его рабочую память превышает ее лимиты.

\section{Методы снижения нагрузки на рабочую память при обучении математике}

1. Автоматизировать базовые вычислительные навыки (арифметические, тригонометрические, геометрические), чтобы процесс вычисления оказывал минимальную нагрузку на рабочую память.

2. Использовать простые предложения при изложении нового материала, поскольку сложносочиненные и сложноподчиненные предложения перегружают слабую рабочую память [11].

3. Указания и инструкции делать краткими, содержащими не более одного условия «если», и относиться они должны непосредственно к текущей задаче. Давать следующую инструкцию можно только после выполнения предыдущей инструкции всеми учениками в классе [11].

4. Справочные материалы для решения задач должны быть на уровне тетради, в которой решается задача: на планшете, смартфоне, в распечатанном виде. Поскольку при переводе взгляда с дальнего объекта (доски) на ближний (тетрадь) может произойти сбой рабочей памяти [11].

5. Математические факты (формулы, правила), часто используемые при решении задач, необходимо заучивать наизусть, поскольку извлечение из долговременной памяти хорошо запомнившейся информации не оказывает нагрузки на рабочую память. Например, формулы приведения. Есть мнемотехническое правило, нагружающее рабочую память. И если заучить часто используемые формулы $(\mathrm{F}(\pi \pm x), \mathrm{F}(\pi / 2 \pm x))$, такую нагрузку можно исключить [12].

6. Минимизировать интенсивность потока новой информации, перераспределив ее во времени. На одном уроке изучаем метод решения иррациональных уравнений, оговорив, что полученные решения надо проверять подстановкой, а причину появления лишних корней и решение иррациональных неравенств оставляем на следующее занятие. То есть разводим по времени изучение правил и исключений из них [12].

7. Наглядно представлять материал в виде концептуальных схем, графов, интеллект-карт. Это не только снижает нагрузку на рабочую память, но и дает более глубокий уровень восприятия новой информации, что, в свою очередь, обеспечивает лучшее запоминание [12].

8. Использовать мнемотехнику для устойчивой консолидации в долговременной памяти новой информации. Хотя мнемотехника применяется в математике не так часто, как при изучении гуманитарных дисциплин, пренебрегать ею не стоит. Например, «правило лошади» в тригонометрии связывает условие замены функции на кофункцию с общепринятыми движениями головы при выражении согласия или несогласия [12].

\section{Использование компьютерных тренажеров}

Необходимое условие обучения неспособных учеников основам элементарной математики - индивидуализация и специально разработанная методика: «Детальное исследование (в течение года) группы неспособных учеников еще раз убедительно показало, что в процессе самостоятельной работы им доступна лишь элементарная степень обобщения мате- 
матического материала. Более высокий уровень обобщения (необходимый для сколько-нибудь удовлетворительного овладения математикой) наступал лишь постепенно, в результате очень большого труда и при прямой помощи экспериментатора. В ряде случаев такое обобщение возникало только в результате специально организованной экспериментатором работы. В трудных случаях обобщение не возникало совсем» [1, с. 274-275].

В результате многолетних исследований математических способностей В. А. Крутецкий делает вывод: «...неспособных учеников нужно... длительно тренировать, упражнять на специально подобранном материале, охватывающем все возможные случаи и комбинации несущественных признаков, чтобы им стала доступной более или менее элементарная степень обобщения» [1, с. 259].

«Длительная тренировка» на специально подобранных упражнениях позволяет преодолеть объективные трудности изучения математики у неспособных учеников по причине того, что автоматизация базовых вычислительных навыков (арифметических, тригонометрических, геометрических) снижает нагрузку на рабочую память при решении соответствующих задач, высвобождая ее ресурсы на осмысление условия и поиск алгоритмов.

Компьютерные программы могут быть генератором таких «специально подобранных упражнений» и одновременно репетитором, подсказывающим порядок их выполнения и оценивающим результат работы. Автором разработана система таких компьютерных тренажеров, доступных по адресу: http://www.workingmemory.ru/.

На рис. 2-5 представлены их скрины, а в табл. 1 дано краткое описание. Ссылка на видео с описанием методики работы с тренажерами есть на главной странице сайта http:// www.workingmemory.ru/htmls/trainers2. Можно бесплатно зарегистрироваться на сайте, чтобы составить объективное представление о работе с тренажерами.

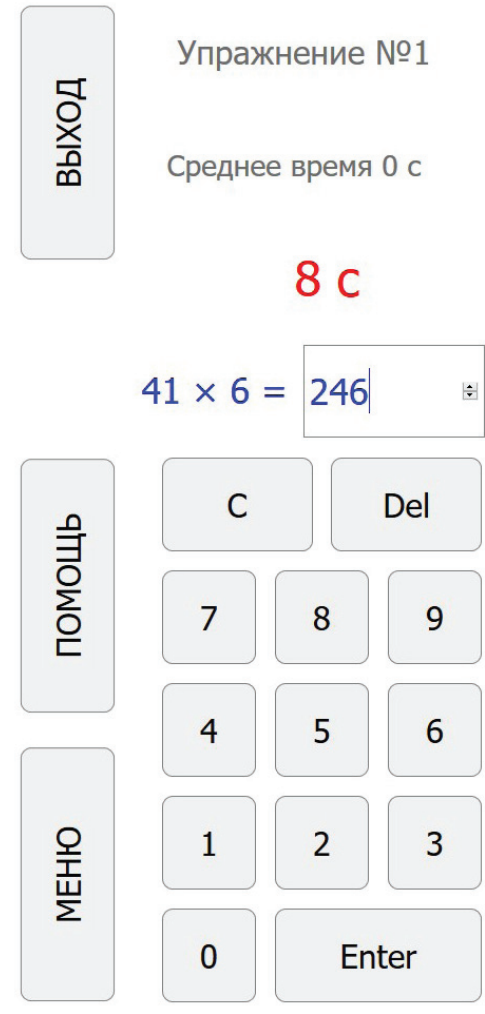

Рис. 2. Скрин тренажера «Устный счет». Оптимизирован для мобильных устройств

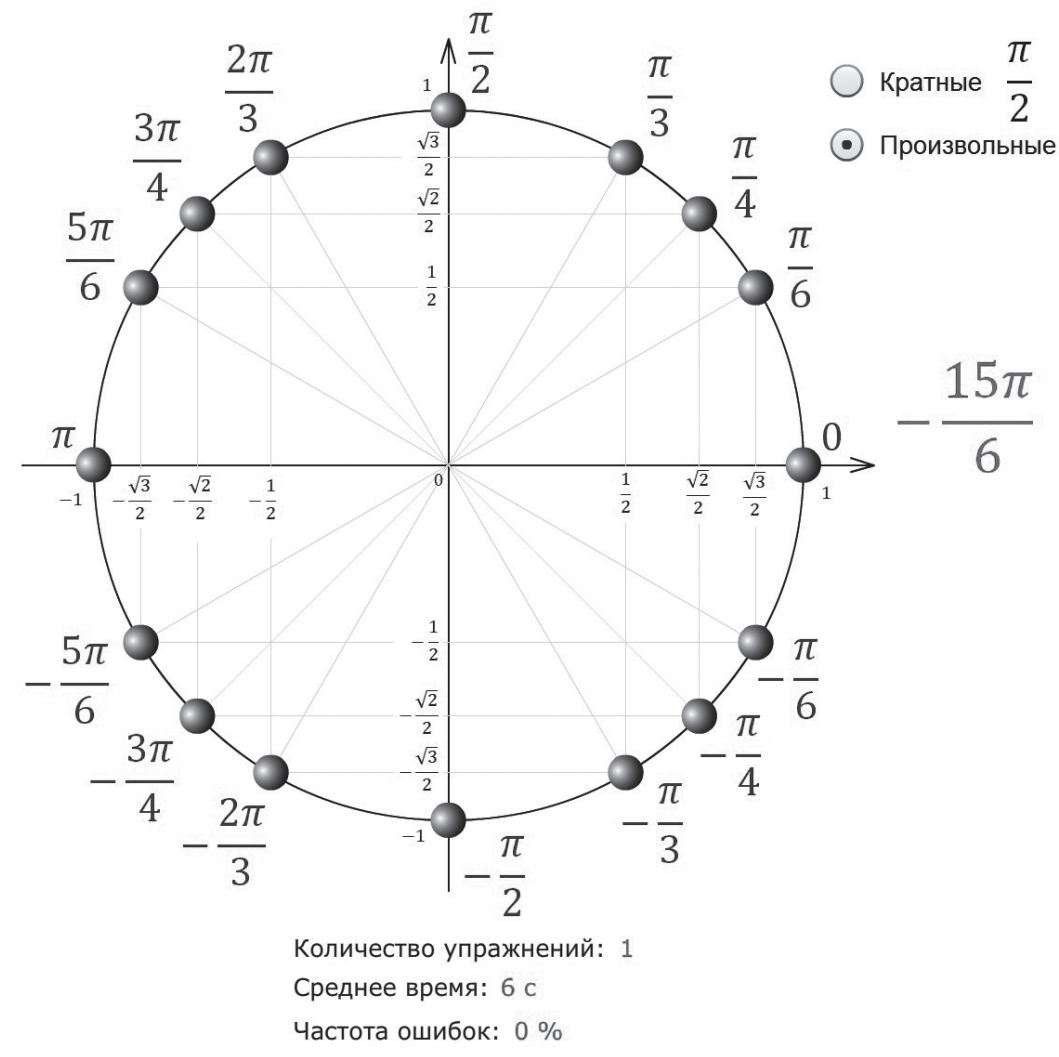

Рис. 3. Скрин тренажера «Тригонометрическая окружность» 


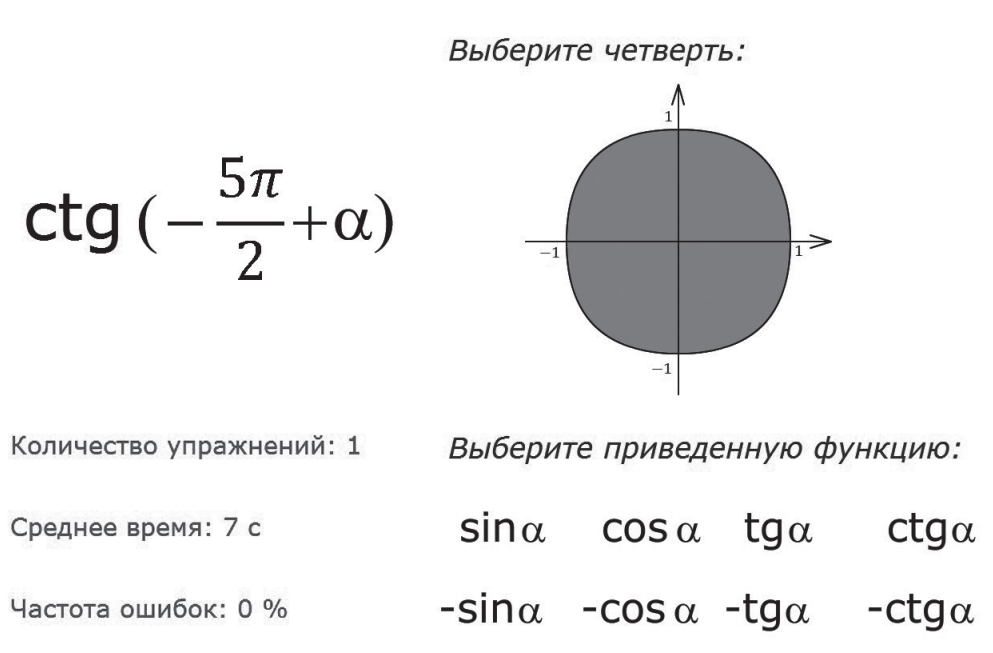

Рис. 4. Скрин тренажера «Формулы приведения»

Рис. 5. Скрин тренажера «Прямоугольный треугольник». Оптимизирован для мобильных устройств

\section{Пример № 1}

АВC - прямоугольный треугольник, СН - высота.

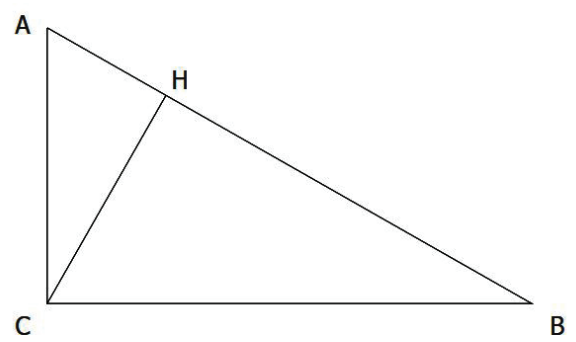

Дано: $\angle \mathrm{A}=45^{\circ}, \mathrm{CH}=9$

Найти: $\mathrm{BC}=$ ?

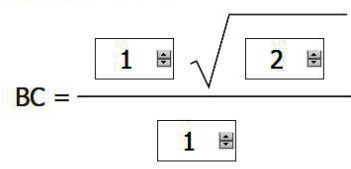

ВыхоД

Таблица 1

Краткое описание компьютерных тренажеров, опубликованных по адресу: http://www.workingmemory.ru/htmls/trainers2

\begin{tabular}{|l|c|c|c|}
\hline \multicolumn{1}{|c|}{$\begin{array}{c}\text { Наименование } \\
\text { тренажера }\end{array}$} & Описание & $\begin{array}{c}\text { Номера задач профиль- } \\
\text { ного ЕГЭ по математике } \\
\text { образца 2020 г. }\end{array}$ & Классы \\
\hline Устный счет & $\begin{array}{c}\text { Развивает навыки устного счета, в том числе с } \\
\text { использованием различных приемов и схем } \\
\text { вычисления }\end{array}$ & $1-19$ & $2-11$ \\
\hline $\begin{array}{l}\text { Тригонометрическая } \\
\text { окружность }\end{array}$ & $\begin{array}{c}\text { Развивает навык быстрого нахождения точки на } \\
\text { числовой окружности для заданного значения } \\
\text { числа в радианах или градусах }\end{array}$ & $5,9,10,13$ & $10-11$ \\
\hline $\begin{array}{l}\text { Формулы приведе- } \\
\text { ния }\end{array}$ & $\begin{array}{c}\text { Развивает навык безошибочного применения } \\
\text { формул приведения в решении тригонометриче- } \\
\text { ских уравнений и неравенств }\end{array}$ & $5,9,13$ & $10-11$ \\
\hline $\begin{array}{l}\text { Прямоугольный } \\
\text { треугольник }\end{array}$ & $\begin{array}{c}\text { Развивает способность к автоматическому и } \\
\text { безошибочному определению отношений между } \\
\text { сторонами прямоугольного треугольника на } \\
\text { основе значений тригонометрических функций } \\
\text { острых углов }\end{array}$ & $6,14,16$ & $8-11$ \\
\hline
\end{tabular}

\section{Методика работы с тренажерами}

1. Последовательное изучение базовых правил выполнения вычислительных операций (арифметических, тригонометрических или геометрических).

2. Ежедневная тренировка по 20 минут в течение месяца. Отслеживание среднего времени выполнения упражнения и частоты ошибок позволяет увидеть положительную динамику. Ученик субъективно чувствует, что вычислительные задачи решаются легче, быстрее и без ошибок.

3. Перерыв в течение месяца и повторные тренировки для устойчивого закрепления вычислительных навыков в долговременной памяти.

Участники эксперимента отбирались из числа слушателей курсов по подготовке к ЕГЭ по математике (10-11-й класс). Каждый участник получил уникальный логин и пароль. Результаты каждого упражнения сохранялись в виде отдельной записи в базе данных на сер- 
вере. Запись включает уникальный логин учащегося. На момент написания статьи в эксперименте участвовал 31 старшеклассник.

В результате тренировок заметно уменьшилось среднее время на выполнение одного упражнения (табл. 2) и снизилась частота ошибок (табл. 3). Сокращение времени на одно упражнение свидетельствует об уменьшении нагрузки на рабочую память, а снижение частоты ошибок о более эффективном использовании ее ресурсов.

Эффект от тренировок характеризуют отзывы самих учащихся как во время обучения на курсах («Благодаря тренажерам я получил в школе пять на уроке, потому что лучше всех решал задачи по тригонометрии»), так и после сдачи ЕГЭ по математике («Я не допустил ни одной ошибки во второй части ЕГЭ...»). Данные тренажеры представляют собой не только инструмент обучения неспособных школьников, но и ноу-хау успешной подготовки к государственному экзамену по математике.

Таблица 2

Среднее время на выполнение одного упражнения с учетом

среднеквадратичного отклонения

\begin{tabular}{|l|c|c|}
\hline \multicolumn{1}{|c|}{ Тренажер } & \multicolumn{2}{c|}{ Среднее время на выполнение одного упражнения, с } \\
\cline { 2 - 3 } & В начале курса тренировок & После курса тренировок \\
\hline Тригонометрическая окружность & $35 \pm 20$ & $13 \pm 11$ \\
\hline Формулы приведения & $33 \pm 17$ & $21 \pm 5$ \\
\hline $\begin{array}{l}\text { Устный счет. Упражнения, в которых одно } \\
\text { из чисел однозначное, а другое - двузначное }\end{array}$ & $18 \pm 7$ \\
\hline $\begin{array}{l}\text { Устный счет. Упражнение на произведение } \\
\text { двухзначных чисел в уме }\end{array}$ & $57 \pm 27$ & $20 \pm 11$ \\
\hline
\end{tabular}

Таблица 3

Доля ошибок от числа правильно выполненных упражнений с учетом

среднеквадратичного отклонения

\begin{tabular}{|l|c|c|}
\hline \multirow{2}{*}{\multicolumn{1}{|c|}{ Тренажер }} & \multicolumn{2}{|c|}{ Количество ошибок, \% } \\
\cline { 2 - 3 } & В начале курса тренировок & После курса тренировок \\
\hline Тригонометрическая окружность & $54 \pm 48$ & $10 \pm 8$ \\
\hline Формулы приведения & $52 \pm 32$ & $29 \pm 14$ \\
\hline
\end{tabular}

Предельные значения показателей работы с тренажерами зависят как от рабочей памяти ученика, так и от прилагаемых им усилий. Поэтому средние отклонения показателей сравнимы со средними значениями в табл. 2 и 3.

Наличие в тренажерах счетчиков времени и вычислительных ошибок переводит рабочую память школьника в режим предельной нагрузки, что косвенным образом способствует ее улучшению путем тренировки. Количественно этот эффект не был оценен, поскольку рабочая память учащихся не измерялась. Однако его следует иметь в виду как один из аргументов о пользе применения подобных тренажеров в учебном процессе.

\section{Заключение}

Достичь наилучшего результата в обучении неспособных школьников математике можно, если исключить перегрузку их рабочей памяти. Один из методов - развитие их вычислительных навыков с помощью компьютерных тренажеров, разработанных автором. Это повышает эффективность использования ими рабочей памяти, высвобождает ее ресурсы для овладения методами решения задач и усвоения новых знаний. Таким образом, повышается успеваемость неспособных школьников при изучении базового курса школьной математики. 


\section{Список литературы}

1. Крутецкий В. А. Психология математических способностей школьников / под ред. Н. И. Чуприковой. М.: Институт практической психологии; Воронеж: МОДЭК, 1998. 416 с.

2. Baddeley A. Working memory: Looking back and looking forward // Nature Reviews. 2003. Vol. 4. P. 829-839. DOI: 10.1038/ nrn1201.

3. Alloway T. P., Passolunghi M. C. The relationship between working memory, IQ and mathematical skills in children // Learning and Individual Differences. 2011. Vol. 21 (1). P. 133-137. DOI: 10.1016/j.lindif.2010.09.013.

4. Alloway T. P. How does working memory work in the classroom? // Educational Research and Reviews. 2006. № 1 (4). P. 134-139.

5. Berg D. H. Working memory and arithmetic calculation in children: The contributory roles of processing speed, shortterm memory, and reading // Journal of Experimental Child Psychology. 2008. Vol. 99 (4). P. 288-308. DOI: 10.1016/j.jecp.2007.12.002.

6. Andersson U. The contribution of working memory to children's mathematical word problem solving // Applied Cognitive Psychology. 2007. Vol. 21. P. 1201-1216. DO I: 10.1002/acp.1317.

7. Milton J. Dehn. Working Memory and Academic Learning: Assessment and Intervention. Wiley \& Sons, 2008. 386 p.

8. Jaeggi S. M., Buschkuehl M., Jonides J., Perrig W. J. Improving fluid intelligence with training on working memory // PNAS. 2008. Vol. 105, № 19. P. 6829-6833. DOI: $10.1073 /$ pnas.0801268105.

9. Dunning D. L., Holmes J., Gathercole S. E. Does working memory training lead to generalized improvements in children with low working memory? A randomized controlled trial // Developmental Science. 2013. Vol. 16 (6). P. 915-925. DOI: 10.1111/ desc.12068.

10. Sánchez-Pérez N., Castillo A., López-López J. A., Pina V., Puga J. L., Campoy G., González-Salinas C., Fuentes L. J. Computer-Based Training in Math and Working Memory Improves Cognitive Skills and Academic Achievement in Primary School Children: Behavioral Results // Frontiers in Psychology. 2018. Jan 9. V. 8, № 2327. Doi: 10.3389/fpsyg.2017.02327.

11. Gathercole S. E., Alloway T. P. Working Memory and Learning: A Practical Guide for Teachers. SAGE Publications, 2008. $128 \mathrm{p}$.

12. Watson A. C. Learning Begins: The Science of Working Memory and Attention for the Classroom Teacher (A Teacher's Guide to the Learning Brain). Rowman \& Littlefield, 2017. 174 p.

Пигарев Александр Юрьевич, кандидат педагогических наук, доцент, Новосибирский государственный университет экономики и управления (ул. Каменская, 56, Новосибирск, Россия, 630099). E-mail: physflash@yandex.ru

Материал поступил в редакиию 12.11.2019.

DOI 10.23951/2307-6127-2020-1-37-45

\section{METHODOLOGICAL ASPECTS OF TEACHING MATHEMATICS TO INCAPABLE STUDENTS}

\section{A. Yu. Pigarev}

\section{Novosibirsk State University of Economics and Management, Novosibirsk, Russian Federation}

Differences in the mathematical abilities of different students are manifested in the fact that the same practice and exercises for students with different abilities give different results. For a capable student, these exercises lead to the mastery of mathematical knowledge and skills, for an incapable student do not. The reason for the inability to mathematics is the lack of working memory. There are two main approaches to solve the problem of teaching mathematics to incapable students: (1) training working memory and (2) reducing the load on working memory in the educational process. The results of the first approach are ambiguous: training working memory leads to an improvement in the performance of untrained tests for working memory, but it may not lead to noticeable changes in learning indicators associated with the working memory of the student. 
Accordingly, it remains to reduce the load on working memory in the educational process. Well known methods that reduce the load on working memory when studying mathematics are described.

Automation of basic computing skills (arithmetic, trigonometric, geometric) is achieved with the help of computer trainers developed by the author: the mental calculations trainer, the trainer for developing skills in working with a trigonometric circle, the trainer for developing skills of using reduction formulas and the rectangular triangle solving trainer. The technique of working with trainers is based on the interval repetition method. Empirical data on the results of their implementation are presented.

Keywords: mathematical abilities, teaching mathematics, working memory, computer trainer, interval repetition.

\section{References}

1. Krutetskiy V. A. Psikhologiya matematicheskikh sposobnostey shkol'nikov. Pod red. N. I. Chuprikovoy [Psychology of mathematical abilities of schoolchildren. Ed. N.I. Chuprikova]. Moscow, Institute of Practical Psychology Publ.; Voronezh, MODEK Publ., 1998. 416 p. (in Russian).

2. Baddeley A. Working memory: Looking back and looking forward. Nature Reviews, 2003, vol. 4, pp. 829-839. DOI: 10.1038/ nrn1201.

3. Alloway T. P., Passolunghi M. C. The relationship between working memory, IQ and mathematical skills in children. Learning and Individual Differences, 2011, vol. 21 (1), pp. 133-137. DOI: 10.1016/j.lindif.2010.09.013.

4. Alloway T. P. How does working memory work in the classroom? Educational Research and Reviews, 2006, no. 1 (4), pp. 134-139.

5. Berg D. H. Working memory and arithmetic calculation in children: The contributory roles of processing speed, shortterm memory, and reading. Journal of Experimental Child Psychology, 2008, vol. 99 (4), pp. 288-308. DOI: 10.1016/j.jecp.2007.12.002.

6. Andersson U. The contribution of working memory to children's mathematical word problem solving. Applied Cognitive Psychology, 2007, vol. 21, pp. 1201-1216. DOl: 10.1002/acp.1317.

7. Milton J. Dehn. Working Memory and Academic Learning: Assessment and Intervention. Wiley \& Sons, 2008. 386 p.

8. Jaeggi S. M., Buschkuehl M., Jonides J., Perrig W. J. Improving fluid intelligence with training on working memory. PNAS, 2008, vol. 105, no. 19, pp. 6829-6833. DOl: 10.1073/pnas.0801268105.

9. Dunning D. L., Holmes J., \& Gathercole S. E. Does working memory training lead to generalized improvements in children with low working memory? A randomized controlled trial. Developmental Science, 2013, vol. 16 (6), pp. 915-925. DOI: 10.1111/ desc.12068.

10. Sánchez-Pérez N., Castillo A., López-López J. A., Pina V., Puga J. L., Campoy G., González-Salinas C., Fuentes L. J. Computer-Based Training in Math and Working Memory Improves Cognitive Skills and Academic Achievement in Primary School Children: Behavioral Results. Frontiers in Psychology, 2018, Jan 9, vol. 8, no. 2327. Doi: 10.3389/fpsyg.2017.02327.

11. Gathercole S. E., Alloway T. P. Working Memory and Learning: A Practical Guide for Teachers. SAGE Publications, 2008. $128 \mathrm{p}$.

12. Watson A. C. Learning Begins: The Science of Working Memory and Attention for the Classroom Teacher (A Teacher's Guide to the Learning Brain). Rowman \& Littlefield, 2017. 174 p.

Pigarev A. Yu., Novosibirsk State University of Economics and Management (ul. Kamenskaya, 56, Novosibirsk, Russian Federation, 630099). E-mail: physflash@yandex.ru 